\title{
RELATIVE MODULAR UNIFORM APPROXIMATION BY MEANS OF THE POWER SERIES METHOD WITH APPLICATIONS
}

\author{
KULDIP RAJ AND ANU CHOUDHARY
}

\begin{abstract}
We introduce the notion of relative convergence by means of a four dimensional matrix in the sense of the power series method, which includes Abel's as well as Borel's methods, to prove a Korovkin type approximation theorem by using the test functions $\left\{1, y, z, y^{2}+z^{2}\right\}$ and a double sequence of positive linear operators defined on modular spaces. We also endeavor to examine some applications related to this new type of approximation.
\end{abstract}

\section{INTRODUCTION AND PRELIMINARIES}

Korovkin type approximation plays an important role in summability theory. The classical Bohman-Korovkin theorem establishes the uniform convergence in the space $C[a, b]$ of all continuous real valued functions defined on the interval $[a, b]$, for a sequence of positive linear operators acting on $C[a, b]$ assuming the convergence only on the test functions $1, x$, and $x^{2}$. In case of the space of continuous $2 \pi$-periodic real valued functions the convergence is considered on the test functions $1, \cos x$, and $\sin x$. In 1953, Korovkin discovered this property, which is a now a matter of interest for several mathematicians. Many mathematicians have worked on generalizing the Korovkin theorems in several ways. Initially, Bardaro and Mantellini [3] obtained some approximation theorems by means of positive linear operators defined on modular spaces. Later on, they studied the classical Korovkin theorem in multivariate modular function spaces 44. In [15] Karakuş and Demirci used matrix summability to obtain approximation results on modular spaces. The statistical version of Korovkin's theorem has been presented by Mursaleen and Alotaibi in [21] and Belen et al. in [7]. In summability theory the power series method of convergence is very prominent as it includes both Abel's and Borel's methods of convergence. From the existing literature we found that there is no comparison between statistical $A$-approximation process and the power series method. Since these two methods of convergence are totally different, it is clear from the literature study that there is no particular matrix $A$ such that the corresponding statistical method generalizes the power series method. These two

2010 Mathematics Subject Classification. 40A05; 40A30.

Key words and phrases. Modular space; power series; relative convergence; double sequence space; positive linear operator. 
methods have their own importance and are independent of each other. Most of the authors studied these two methods independently. Orhan and Demirci [23] studied the concept of statistical $A$-summability in modular spaces. In [22] they studied the problem of approximation to a function by means of a double sequence of positive linear operators on modular spaces by using the concept of statistical convergence. In [2], Bardaro et al. proved a Korovkin type approximation theorem with respect to $\Psi$ - $A$-statistical convergence. Recently, Yilmaz et al. 30] defined a new type of modular convergence by using the notion of relative convergence and proved some results on the approximation theorems. Further, Demirci and Orhan [12] studied the statistical relative approximation problem by means of double sequence of positive linear operator defined on modular spaces. Yurdakdim [31] defined power series modular convergence and studied Korovkin-type approximation results. In [28] Tas discussed some results concerning Mastroianni operators by the power series method. In this paper, we study the problem of approximation to a function using a double sequence of positive linear operators on a modular space by means of relative convergence, four dimensional regular summability matrix method, and the concept of power series convergence which is stronger than the Pringsheim convergence.

Many other versions of the Korovkin theorem have been studied by several authors. Likewise, Bardaro et al. [6] studied some version of abstract Korovkin type theorem on modular spaces with respect to the filter convergence on positive linear operators. Mursaleen and Mohiuddine [20] proved the Korovkin type approximation theorem with respect to almost convergence and statistical convergence by using the test functions $\left\{1, e^{-x}, e^{-2 x}\right\}$. Alotaibi et al. [1] used the notion of $\sigma$ convergence of double sequences to prove an approximation theorem.

We now recall some basic definitions and notations used in this paper.

A double sequence $x=\left(x_{k, l}\right)$ has Pringsheim limit $L$ (denoted by $P-\lim x=L$ ) provided that given $\epsilon>0$ there exists $K \in \mathbb{N}$ such that $\left|x_{k, l}-L\right|<\epsilon$ whenever $k, l>K$ (see [24]). A double sequence $x=\left(x_{k, l}\right)$ is bounded if there exists a positive number $K$ such that $\left|x_{k, l}\right|<K$ for all $k$ and $l$. Some initial work on double sequences is due to Bromwich [8]. Later on, the double sequences were studied in [14, 18, 19, 25, 26, 29.

Let $q_{i, j}$ be a double sequence of non-negative numbers with $q_{00}>0$ and such that the power series

$$
q(c, d)=\sum_{i, j=0}^{\infty} q_{i, j} c^{i} d^{j}
$$

has radius of convergence $R \in(0, \infty]$ with $c, d \in(0, R]$. Let $x=\left(x_{i, j}\right)$ be a double sequence of real numbers. If

$$
\lim _{c, d \rightarrow R^{-}} \frac{1}{q(c, d)} \sum_{i, j=0}^{\infty} q_{i, j} c^{i} d^{j} x_{i, j}=L
$$

for all $c, d \in(0, R)$, then $x=\left(x_{i, j}\right)$ is said to be convergent to $L$ in the sense of the power series method. 
Example 1.1. Let

$$
x=\left\{x_{i, j}\right\}= \begin{cases}0, & j \text { is not a multiple of } 3, \\ 1, & \text { otherwise }\end{cases}
$$

$q_{i, j}=1 \forall i, j \in \mathbb{N},|c|<1$, and $|d|<1$. So we get

$$
q(c, d)=\sum_{i, j=0}^{\infty} c^{i} d^{j}=\sum_{i=0}^{\infty} c^{i} \sum_{j=0}^{\infty} d^{j}=\frac{1}{1-d} \sum_{i=0}^{\infty} c^{i}=\frac{1}{1-d} \frac{1}{1-c} .
$$

Then

$$
\begin{aligned}
\lim _{c, d \rightarrow 1^{-}} \frac{1}{q(c, d)} \sum_{i, j=0}^{\infty} q_{i j} c^{i} d^{j} x_{i j} & =\lim _{c, d \rightarrow 1^{-}}(1-d)(1-c) \sum_{i=0}^{\infty} c^{i} \sum_{j=0}^{\infty} d^{3 j} \\
& =\lim _{c, d \rightarrow 1^{-}}(1-d)(1-c) \frac{1}{1-d^{3}} \sum_{i=0}^{\infty} c^{i} \\
& =\lim _{c, d \rightarrow 1^{-}} \frac{(1-d)(1-c)}{\left(1-d^{3}\right)(1-c)} \\
& =\frac{1}{3} .
\end{aligned}
$$

So the given double sequence is convergent to $\frac{1}{3}$ in the sense of the power series method but it is not convergent in the Pringsheim sense.

By the above example one can easily say that the power series method is stronger than ordinary convergence. If for any non-negative integers $\alpha, \beta$ one has that

$$
\lim _{c, d \rightarrow R^{-}} \frac{\sum_{i=0}^{\infty} q_{i, \alpha} c^{i}}{q_{(c, d)}}=0 \text { and } \lim _{c, d \rightarrow R^{-}} \frac{\sum_{j=0}^{\infty} q_{\beta, j} d^{j}}{q_{(c, d)}}=0,
$$

then the power series method for double sequences is said to be regular.

Let $H=[k, l]$ be a bounded interval of the real line $\mathbb{R}$ provided with Lebesgue measure. Let $C\left(H^{2}\right)$ denote the space of all continuous real valued functions on $H^{2}=[k, l] \times[k, l]$. By $X\left(H^{2}\right)$ we denote the space of all real valued measurable functions on $H^{2}$ with equality almost everywhere, and by $C^{\infty}\left(H^{2}\right)$ we denote the space of all infinitely differentiable functions on $H^{2}$.

The concept of uniform convergence of a sequence of functions relative to a scale function was introduced by Moore [17]. Then, Chittenden [10] defined it as follows:

A sequence $\left(f_{i}\right)$ of functions defined on an interval $H=(a \leq x \leq b)$ is said to converge relatively to a limit function $f$, if there exists a scale function $\sigma(x)$ such that for every $\epsilon>0$

$$
\left|f_{i}(x)-f(x)\right|<\epsilon|\sigma(x)|
$$

holds uniformly in $x$ on the interval $H$ for every $i>i_{\epsilon}$. Uniform convergence is the special case of relative convergence in which the scale function is a non-zero constant. For more details see [9, 10, 11]. 
A functional $\rho: X\left(H^{2}\right) \rightarrow[0,+\infty]$ is said to be modular on $X\left(H^{2}\right)$ if it satisfies the following conditions:

(i) $\rho(f)=0$ if and only if $f=0$ a.e. in $H^{2}$,

(ii) $\rho(-f)=\rho(f)$ for every $f \in X\left(H^{2}\right)$,

(iii) $\rho(\alpha f+\beta g) \leq \rho(f)+\rho(g)$ for every $f, g \in X\left(H^{2}\right)$ and for any $\alpha, \beta \geq 0$ with $\alpha+\beta=1$.

In 4, 5] Bardaro et al. have given the concept of $Q$-quasi convex and $Q$-quasi semiconvex modular. They proved that if there exists a constant $Q \geq 1$ such that

$$
\rho(\alpha f+\beta g) \leq Q \alpha \rho(Q f)+Q \beta \rho(Q g)
$$

holds for every $f, g \in X\left(H^{2}\right), \alpha, \beta \geq 0$ with $\alpha+\beta=1$, then a modular $\rho$ is $Q$-quasi convex. If $Q=1$ then $\rho$ is called convex. Furthermore, if

$$
\rho(\alpha f) \leq Q \alpha \rho(Q f)
$$

hold for every $f \in X\left(H^{2}\right)$ and $\alpha \in(0,1]$, then $\rho$ is said to be $Q$-quasi semiconvex. From the above definitions one can say that every $Q$-quasi convex modular is $Q$ quasi semiconvex.

Let $A=\left(a_{k l i j}\right)$ be a four dimensional matrix for all $k, l, i, j \in \mathbb{N}$ and $x=\left(x_{i, j}\right)$ be a double sequence. Then the sum

$$
y_{k, l}=\sum_{i, j=1,1}^{\infty, \infty} a_{k l i j} x_{i, j}
$$

is called the $A$-means of the double sequence $\left(x_{i, j}\right)$. A double sequence $\left(x_{i, j}\right)$ is said to be $A$-summable to the limit $L$ if the $A$-means exist for all $k, l$ in the sense of Pringsheim's convergence

$$
P-\lim _{p, q \rightarrow \infty} \sum_{i, j=0,0}^{p, q} a_{k l i j} x_{i, j}=y_{k, l}
$$

and

$$
P-\lim _{k, l \rightarrow \infty} y_{k, l}=L .
$$

A four dimensional matrix $A=\left(a_{k l i j}\right)$ is said to be $R H$-regular or bounded regular (see [13, 27]) if it maps every bounded $P$-convergent sequence into a $P$-convergent sequence with the same $P$-limit. The Robinson-Hamilton conditions state that the four dimensional matrix $A=\left(a_{k l i j}\right)$ is $R H$-regular if and only if

$\left(\mathrm{RH}_{1}\right) P-\lim _{k, l} a_{k l i j}=0$ for each $(i, j) \in \mathbb{N}^{2}$;

$\left(\mathrm{RH}_{2}\right) P-\lim _{k, l} \sum_{i, j \in \mathbb{N}^{2}} a_{k l i j}=1$

$\left(\mathrm{RH}_{3}\right) P-\lim _{k, l} \sum_{i=1}^{\infty}\left|a_{k l i j}\right|=0$ for each $j$;

$\left(\mathrm{RH}_{4}\right) P-\lim _{k, l} \sum_{j=1}^{\infty}\left|a_{k l i j}\right|=0$ for each $i$; 
$\left(\mathrm{RH}_{5}\right) \sum_{(i, j) \in \mathbb{N}^{2}}\left|a_{k l i j}\right|$ is $P$-convergent;

$\left(\mathrm{RH}_{6}\right)$ there exist finite positive integers $r$ and $s$ such that $\sum_{i, j>s}\left|a_{k l i j}\right|<r$ holds for every $(k, l) \in \mathbb{N}^{2}$.

Let us consider vector subspaces $L^{\rho}\left(H^{2}\right)$ and $E^{\rho}\left(H^{2}\right)$ of $X\left(H^{2}\right) . L^{\rho}\left(H^{2}\right)$ is called the modular space generated by $\rho$ and $E^{\rho}\left(H^{2}\right)$ is called the space of finite elements of $L^{\rho}\left(H^{2}\right)$. These are defined as follows:

$$
L^{\rho}\left(H^{2}\right)=\left\{f \in X\left(H^{2}\right): \lim _{\eta \rightarrow 0^{+}} \rho(\eta f)=0\right\}
$$

and

$$
E^{\rho}\left(H^{2}\right)=\left\{f \in L^{\rho}\left(H^{2}\right): \rho(\eta f)<+\infty \text { for all } \eta>0\right\} .
$$

Note that if $\rho$ is $Q$-quasi semiconvex, then the space

$$
\left\{f \in X\left(H^{2}\right): \rho(\eta f)<+\infty \text { for some } \eta>0\right\}
$$

coincides with $L^{\rho}\left(H^{2}\right)$.

A double sequence $\left\{f_{i, j}\right\}$ of functions whose terms belong to $L^{\rho}\left(H^{2}\right)$ is said to be relatively modularly convergent to a function $f \in L^{\rho}\left(H^{2}\right)$, iff there exists a scale function $\sigma(y, z) \in X\left(H^{2}\right),|\sigma(y, z)| \neq 0$, such that

$$
P-\lim _{i, j} \rho\left(\eta_{0}\left(\frac{f_{i, j}-f}{\sigma(y, z)}\right)\right)=0 \quad \text { for some } \eta_{0}>0 .
$$

A double sequence $\left\{f_{i, j}\right\}$ is relatively $F$-norm convergent (relatively strongly convergent) to $f$ iff

$$
P-\lim _{i, j} \rho\left(\eta\left(\frac{f_{i, j}-f}{\sigma(y, z)}\right)\right)=0 \quad \text { for every } \eta>0 .
$$

One can note that 1.2 and $(1.3)$ are equivalent iff the modular $\rho$ satisfies the $\Delta_{2}$ condition, that is, there exists a constant $M>0$ such that

$$
\rho(2 f) \leq M(\rho f)
$$

for every $f \in X\left(H^{2}\right)$. A relative strong convergence of the double sequence $\left\{f_{i, j}\right\}$ to $f$ is equivalent to the condition $P$ - $\lim _{i, j} \rho\left(2^{K} \eta\left(\frac{f_{i, j}-f}{\sigma(y, z)}\right)\right)=0$, for all $K=1,2, \ldots$ and for some $\eta>0$. If $\left\{f_{i, j}\right\}$ is relatively modularly convergent to $f$, then the $\Delta_{2}$ condition implies by induction that

$$
\rho\left(2^{K} \eta\left(\frac{f_{i, j}-f}{\sigma(y, z)}\right)\right) \leq M^{K} \rho\left(\eta\left(\frac{f_{i, j}-f}{\sigma(y, z)}\right)\right) .
$$

Hence, we get $P-\lim _{i, j} \rho\left(2^{K} \eta\left(\frac{f_{i, j}-f}{\sigma(y, z)}\right)\right)=0$.

A double sequence $\left\{f_{i, j}\right\}$ of functions whose terms belong to $L^{\rho}\left(H^{2}\right)$ is said to be relatively modularly convergent to a function $f \in L^{\rho}\left(H^{2}\right)$ in the sense of the 
power series method iff there exists a scale function $\sigma(y, z) \in X\left(H^{2}\right),|\sigma(y, z)| \neq 0$, such that

$$
\lim _{c, d \rightarrow R^{-}} \frac{1}{q(c, d)} \sum_{i, j=0}^{\infty} q_{i, j} c^{i} d^{j} \rho\left(\eta_{0}\left(\frac{f_{i, j}-f}{\sigma(y, z)}\right)\right)=0 \quad \text { for some } \eta_{0}>0 .
$$

A sequence $\left\{f_{i, j}\right\}$ is relatively $F$-norm convergent in the sense of the power series method (relatively strongly convergent in the sense of the power series method) to $f$ iff

$$
\lim _{c, d \rightarrow R^{-}} \frac{1}{q(c, d)} \sum_{i, j=0}^{\infty} q_{i, j} c^{i} d^{j} \rho\left(\eta\left(\frac{f_{i, j}-f}{\sigma(y, z)}\right)\right)=0 \quad \text { for every } \eta>0 .
$$

If a scale function $\sigma(y, z)$ is a non zero constant then the modular convergence in the sense of the power series method is the special case of relative modular convergence in the sense of the power series method.

Several properties of the modular $\rho$ are discussed below:

(a) $\rho$ is finite if $\chi B \in L^{\rho}\left(H^{2}\right)$ whenever $B$ is a measurable subset of $H^{2}$ such that $\mu(B)<\infty$.

(b) $\rho$ is absolutely finite if $\rho$ is finite and for every $\epsilon>0, \eta>0$ there exists a $\delta>0$ such that $\rho(\eta \chi A)<\epsilon$ for any measurable subset $A \subset H^{2}$ with $\mu(A)<\delta$.

(c) $\rho$ is strongly finite if $\chi H^{2} \in E^{\rho}\left(H^{2}\right)$.

(d) $\rho$ is monotone if $\rho(f) \leq \rho(g)$ for $|f| \leq|g|$.

(e) $\rho$ is absolutely continuous if for every $f \in X\left(H^{2}\right)$ with $\rho(f)<+\infty$ there exists $\beta>0$ and for every $\epsilon>0$ there is $\delta>0$ such that $\rho(\beta f \chi A)<\epsilon$ whenever $A$ is any measurable subset of $H^{2}$ with $\mu(A)<\delta$.

(f) If the modular $\rho$ is monotone and finite, then $C\left(H^{2}\right) \subset L^{\rho}\left(H^{2}\right)$.

(g) If the modular $\rho$ is monotone and strongly finite, then $C\left(H^{2}\right) \subset E^{\rho}\left(H^{2}\right)$.

(h) If the modular $\rho$ is monotone, absolutely finite and absolutely continuous, then $\overline{C^{\infty}\left(H^{2}\right)}=L^{\rho}\left(H^{2}\right)$.

When $\rho$ is monotone and finite modular on $X\left(H^{2}\right)$, one can construct a subset $G$ satisfying $C^{\infty}\left(H^{2}\right) \subset G \subset L^{\rho}\left(H^{2}\right)$.

Let $\mathbb{S}=\left\{S_{i, j}\right\}$ be a sequence of positive linear operators from $G$ into $X\left(H^{2}\right), X_{\mathbb{S}} \subset$ $G$ containing $C^{\infty}\left(H^{2}\right), A=\left(a_{k l i j}\right)$ a non-negative $R H$-regular summability matrix method, and $\sigma(y, z) \in X\left(H^{2}\right)$ an unbounded function with $\sigma(y, z) \neq 0$ such that

$$
\limsup _{c, d \rightarrow R^{-}} \frac{1}{q(c, d)} \sum_{i, j=0}^{\infty} q_{i, j} c^{i} d^{j} \rho\left(\eta\left(\frac{\sum_{i, j=1,1}^{\infty, \infty} a_{k l i j} S_{i, j} g-g}{\sigma(y, z)}\right)\right) \leq T \rho(\eta g)
$$

holds for every $g \in X_{\mathbb{S}}, \eta>0$, and for an absolute positive constant $T$. By $S_{i, j}(f ; y, z)$, we denote the value of $S_{i, j} f$ at a point $(y, z) \in H^{2}$.

Throughout the paper the operator $\mathbb{S}=\left\{S_{i, j}\right\}$ fulfills condition (1.1). 


\section{MAIN RESUltS}

Theorem 2.1. Let $\mathbb{S}=\left\{S_{i, j}\right\}$ be a double sequence of positive linear operators from $G$ into $X\left(H^{2}\right)$ satisfying (1.4), $A=\left(a_{k l i j}\right)$ be a non-negative $R H$-regular summability matrix method, $\rho$ a strongly finite, monotone, absolutely continuous, $Q$ quasi-convex modular on $X\left(H^{2}\right)$, and $\sigma_{m}(y, z)$ an unbounded function satisfying $\left|\sigma_{m}(y, z)\right| \geq b_{m}>0$ for $m=0,1,2,3$. Assume that

$\lim _{c, d \rightarrow R^{-}} \frac{1}{q(c, d)} \sum_{i, j=0}^{\infty} q_{i, j} c^{i} d^{j} \rho\left(\eta\left(\frac{\sum_{i, j=1,1}^{\infty, \infty} a_{k l i j} S_{i, j}\left(e_{m}\right)-e_{m}}{\sigma_{m}(y, z)}\right)\right)=0 \quad$ for every $\eta>0$

and $m=0,1,2,3$, where $e_{0}(y, z)=1, e_{1}(y, z)=y, e_{2}(y, z)=z$, and $e_{3}(y, z)=$ $y^{2}+z^{2}$. Now let $f \in L^{\rho}\left(H^{2}\right)$ be such that $f-h \in X_{\mathbb{S}}$ for every $h \in C^{\infty}\left(H^{2}\right)$. Then, we have

$\lim _{c, d \rightarrow R^{-}} \frac{1}{q(c, d)} \sum_{i, j=0}^{\infty} q_{i, j} c^{i} d^{j} \rho\left(\eta_{0}\left(\frac{\sum_{i, j=1,1}^{\infty, \infty} a_{k l i j} S_{i, j} f-f}{\sigma(y, z)}\right)\right)=0 \quad$ for some $\eta_{0}>0$,

where $\sigma(y, z)=\max \left\{\left|\sigma_{m}(y, z)\right| ; m=0,1,2,3\right\}$.

Proof. Let $h \in C\left(H^{2}\right) \cap G$. We first show

$$
\lim _{(c, d) \rightarrow R^{-}} \frac{1}{q(c, d)} \sum_{i, j=0}^{\infty} q_{i, j} c^{i} d^{j} \rho\left(\gamma\left(\frac{\sum_{i, j=1,1}^{\infty, \infty} a_{k l i j} S_{i, j} h-h}{\sigma(y, z)}\right)\right)=0 \quad \text { for some } \gamma>0 .
$$

Since $h$ is continuous, as $h \in C\left(H^{2}\right) \cap G$, for given $\epsilon>0$ there exists a number $N>0$ such that for all $(v, w),(y, z) \in H^{2}$ satisfiying $|v-y|<N$ and $|w-z|<N$ we have

$$
|h(v, w)-h(y, z)|<\epsilon .
$$

Also one can see that for all $(v, w),(y, z) \in H^{2}$ satisfying $|v-y|>N$ and $|w-z|>N$ and $\nu=\sup _{(y, z) \in H^{2}}|h(y, z)|$, we have

$$
|h(v, w)-h(y, z)| \leq \frac{2 \nu}{N^{2}}\left\{(v-y)^{2}+(w-z)^{2}\right\} .
$$

Now from 2.4 and 2.5, we have

$$
|h(v, w)-h(y, z)|<\epsilon+\frac{2 \nu}{N^{2}}\left\{(v-y)^{2}+(w-z)^{2}\right\} .
$$

This implies

$$
-\epsilon-\frac{2 \nu}{N^{2}}\left\{(v-y)^{2}+(w-z)^{2}\right\}<h(v, w)-h(y, z)<\epsilon+\frac{2 \nu}{N^{2}}\left\{(v-y)^{2}+(w-z)^{2}\right\} .
$$


Since $\left\{S_{i, j}\right\}$ is a sequence of positive linear operators, by applying $\sum_{i, j=1,1}^{\infty, \infty} a_{k l i j} S_{i, j}$ to 2.6], for every $i, j \in \mathbb{N}$, we have

$$
\begin{aligned}
-\epsilon & \sum_{i, j=1,1}^{\infty, \infty} a_{k l i j} S_{i, j}\left(e_{0} ; y, z\right)-\frac{2 \nu}{N^{2}} \sum_{i, j=1,1}^{\infty, \infty} a_{k l i j} S_{i, j}\left((v-y)^{2}+(w-z)^{2} ; y, z\right) \\
& <\sum_{i, j=1,1}^{\infty, \infty} a_{k l i j} S_{i, j}(h ; y, z)-h(y, z) \sum_{i, j=1,1}^{\infty, \infty} a_{k l i j} S_{i, j}\left(e_{0} ; y, z\right) \\
& <\epsilon \sum_{i, j=1,1}^{\infty, \infty} a_{k l i j} S_{i, j}\left(e_{0} ; y, z\right)+\frac{2 \nu}{N^{2}} \sum_{i, j=1,1}^{\infty, \infty} a_{k l i j} S_{i, j}\left((v-y)^{2}+(w-z)^{2} ; y, z\right) .
\end{aligned}
$$

Hence,

$$
\begin{aligned}
\left|\sum_{i, j=1,1}^{\infty, \infty} a_{k l i j} S_{i, j}(h ; y, z)-h(y, z)\right| \\
\leq\left|\sum_{i, j=1,1}^{\infty, \infty} a_{k l i j} S_{i, j}(h ; y, z)-h(y, z) \sum_{i, j=1,1}^{\infty, \infty} a_{k l i j} S_{i, j}\left(e_{0} ; y, z\right)\right| \\
+\left|h(y, z) \sum_{i, j=1,1}^{\infty, \infty} a_{k l i j} S_{i, j}\left(e_{0} ; y, z\right)-h(y, z)\right| \\
\leq \epsilon \sum_{i, j=1,1}^{\infty, \infty} a_{k l i j} S_{i, j}\left(e_{0} ; y, z\right)+\nu\left|\sum_{i, j=1,1}^{\infty, \infty} a_{k l i j} S_{i, j}\left(e_{0} ; y, z\right)-e_{0}(y, z)\right| \\
\quad+\frac{2 \nu}{N^{2}} \sum_{i, j=1,1}^{\infty, \infty} a_{k l i j} S_{i, j}\left((v-y)^{2}+(w-z)^{2} ; y, z\right) .
\end{aligned}
$$

Thus, for $J=\max \{|y|,|z|\}$ we have

$$
\begin{aligned}
&\left|\sum_{i, j=1,1}^{\infty, \infty} a_{k l i j} S_{i, j}(h ; y, z)-h(y, z)\right| \\
& \leq+\left\{\epsilon+\nu+\frac{4 \nu}{N^{2}} J^{2}\right\}\left|\sum_{i, j=1,1}^{\infty, \infty} a_{k l i j} S_{i, j}\left(e_{0} ; y, z\right)-e_{0}(y, z)\right| \\
&+\frac{4 \nu}{N^{2}} J\left|\sum_{i, j=1,1}^{\infty, \infty} a_{k l i j} S_{i, j}\left(e_{1} ; y, z\right)-e_{1}(y, z)\right| \\
&+\frac{4 \nu}{N^{2}} J\left|\sum_{i, j=1,1}^{\infty, \infty} a_{k l i j} S_{i, j}\left(e_{2} ; y, z\right)-e_{2}(y, z)\right| \\
&+\frac{2 \nu}{N^{2}}\left|\sum_{i, j=1,1}^{\infty, \infty} a_{k l i j} S_{i, j}\left(e_{3} ; y, z\right)-e_{3}(y, z)\right|
\end{aligned}
$$


So by multiplying both sides of the above inequality by $\frac{1}{|\sigma(y, z)|}$ and for any $\gamma>0$, we have

$$
\begin{aligned}
& \gamma\left|\frac{\sum_{i, j=1,1}^{\infty, \infty} a_{k l i j} S_{i, j}(h ; y, z)-h(y, z)}{\sigma(y, z)}\right| \\
& \leq \frac{\gamma \epsilon}{|\sigma(y, z)|}+\operatorname{P\gamma }\left\{\left|\frac{\sum_{i, j=1,1}^{\infty, \infty} a_{k l i j} S_{i, j}\left(e_{0} ; y, z\right)-e_{0}(y, z)}{\sigma(y, z)}\right|\right\} \\
& +\left|\frac{\sum_{i, j=1,1}^{\infty, \infty} a_{k l i j} S_{i, j}\left(e_{1} ; y, z\right)-e_{1}(y, z)}{\sigma(y, z)}\right| \\
& +\left|\frac{\sum_{i, j=1,1}^{\infty, \infty} a_{k l i j} S_{i, j}\left(e_{2} ; y, z\right)-e_{2}(y, z)}{\sigma(y, z)}\right| \\
& +\left|\frac{\sum_{i, j=1,1}^{\infty, \infty} a_{k l i j} S_{i, j}\left(e_{3} ; y, z\right)-e_{3}(y, z)}{\sigma(y, z)}\right|,
\end{aligned}
$$

where $P=\max \left\{\epsilon+\nu+\frac{4 \nu}{N^{2}} J^{2}, \frac{4 \nu}{N^{2}} J, \frac{2 \nu}{N^{2}}\right\}$. Now we apply the modular $\rho$ to both sides of the above inequality. Since $\sigma(y, z)=\max \left\{\left|\sigma_{m}(y, z)\right| ; m=0,1,2,3\right\}$ and $\rho$ is monotone,

$$
\begin{aligned}
\rho\left(\gamma\left(\frac{\sum_{i, j=1,1}^{\infty, \infty} a_{k l i j} S_{i, j} h-h}{\sigma(y, z)}\right)\right) \leq & \rho\left(\gamma \frac{\epsilon}{|\sigma(y, z)|}+\gamma P\left|\frac{\sum_{i, j=1,1}^{\infty, \infty} a_{k l i j} S_{i, j} e_{0}-e_{0}}{\sigma_{0}(y, z)}\right|\right. \\
& +\gamma P\left|\frac{\sum_{i, j=1,1}^{\infty, \infty} a_{k l i j} S_{i, j} e_{1}-e_{1}}{\sigma_{1}(y, z)}\right| \\
& +\gamma P\left|\frac{\sum_{i, j=1,1}^{\infty, \infty} a_{k l i j} S_{i, j} e_{2}-e_{2}}{\sum_{2}^{\infty, \infty} a_{k l i j}(y, z)}\right| \\
& \left.+\gamma P\left|\frac{\left|\frac{i, j=1,1}{i_{i, j} e_{3}-e_{3}}\right|}{\sigma_{3}(y, z)}\right|\right) .
\end{aligned}
$$


Thus, we can write

$$
\begin{aligned}
\rho\left(\gamma\left(\frac{\sum_{i, j=1,1}^{\infty, \infty} a_{k l i j} S_{i, j} h-h}{\sigma(y, z)}\right)\right) \leq & \rho\left(\frac{5 \gamma \epsilon}{\sigma(y, z)}\right) \\
& +\rho\left(5 \gamma P\left(\frac{\sum_{i, j=1,1}^{\infty, \infty} a_{k l i j} S_{i, j} e_{0}-e_{0}}{\sigma_{0}(y, z)}\right)\right) \\
& +\rho\left(5 \gamma P\left(\frac{\sum_{i, j=1,1}^{\infty, \infty} a_{k l i j} S_{i, j} e_{1}-e_{1}}{\sigma_{1}(y, z)}\right)\right) \\
& +\rho\left(5 \gamma P\left(\frac{\sum_{i, j=1,1}^{\infty, \infty} a_{k l i j} S_{i, j} e_{2}-e_{2}}{\sum_{2}(y, z)}\right)\right) \\
& +\rho\left(5 \gamma P\left(\frac{\sum_{i, j=1,1}^{\infty, \infty} a_{k l i j} S_{i, j} e_{3}-e_{3}}{\sigma_{3}(y, z)}\right)\right)
\end{aligned}
$$

Since $\rho$ is $Q$-quasi convex and strongly finite, for $\epsilon \in(0,1]$ we have

$$
\begin{aligned}
\rho\left(\gamma\left(\frac{\sum_{i, j=1,1}^{\infty, \infty} a_{k l i j} S_{i, j} h-h}{\sigma(y, z)}\right)\right) \leq & Q \epsilon \rho\left(\frac{5 \gamma Q}{\sigma(y, z)}\right) \\
& +\rho\left(5 \gamma P\left(\frac{\sum_{i, j=1,1}^{\infty, \infty} a_{k l i j} S_{i, j} e_{0}-e_{0}}{\sigma_{0}(y, z)}\right)\right) \\
& +\rho\left(5 \gamma P\left(\frac{\sum_{i, j=1,1}^{\infty, \infty} a_{k l i j} S_{i, j} e_{1}-e_{1}}{\sum_{1}(y, z)}\right)\right) \\
& +\rho\left(5 \gamma P\left(\frac{\sum_{i, j=1,1}^{\infty, \infty} a_{k l i j} S_{i, j} e_{2}-e_{2}}{\sum_{2}(y, z)}\right)\right) \\
& +\rho\left(5 \gamma P\left(\frac{\sum_{i, j=1,1}^{\infty, \infty} a_{k l i j} S_{i, j} e_{3}-e_{3}}{\sigma_{3}(y, z)}\right)\right)
\end{aligned}
$$


Without loss of generality for $\epsilon \in(0,1]$, one can write

$$
\begin{aligned}
\frac{1}{q(c, d)} \sum_{i, j=0}^{\infty} q_{i, j} c^{i} d^{j} \rho\left[\gamma\left(\frac{\sum_{i, j=1,1}^{\infty, \infty} a_{k l i j} S_{i, j} h-h}{\sigma(y, z)}\right)\right] \\
\leq Q \epsilon \rho\left(\frac{5 \gamma Q}{\sigma(y, z)}\right)+\frac{1}{q(c, d)} \sum_{i, j=0}^{\infty} q_{i, j} c^{i} d^{j} \rho\left[5 \gamma P\left(\frac{\sum_{i, j=1,1}^{\infty, \infty} a_{k l i j} S_{i, j} e_{0}-e_{0}}{\sum_{0}(y, z)}\right)\right] \\
+\frac{1}{q(c, d)} \sum_{i, j=0}^{\infty} q_{i, j} c^{i} d^{j} \rho\left[5 \gamma P\left(\frac{\sum_{i, j=1,1}^{\infty} a_{k l i j} S_{i, j} e_{1}-e_{1}}{\sigma_{1}(y, z)}\right)\right] \\
+\frac{1}{q(c, d)} \sum_{i, j=0}^{\infty} q_{i, j} c^{i} d^{j} \rho\left[5 \gamma P\left(\frac{\sum_{i, j=1,1}^{\infty, \infty} a_{k l i j} S_{i, j} e_{2}-e_{2}}{\sum_{2}(y, z)}\right)\right] \\
+\frac{1}{q(c, d)} \sum_{i, j=0}^{\infty} a_{k l i j} s_{i, j} e_{3}-e_{3} \\
\sum^{i} d^{j} \rho\left[5 \gamma P\left(\frac{i, j=1,1}{\sigma_{3}(y, z)}\right)\right]
\end{aligned}
$$

Now taking limit as $c, d \rightarrow R^{-}$to both sides and by using hypothesis 2.1 , we get

$$
\lim _{c, d \rightarrow R^{-}} \frac{1}{q(c, d)} \sum_{i, j=0}^{\infty} q_{i, j} c^{i} d^{j} \rho\left(\gamma\left(\frac{\sum_{i, j=1,1}^{\infty, \infty} a_{k l i j} S_{i, j} h-h}{\sigma(y, z)}\right)\right)=0 .
$$

Clearly (2.3) holds for every $h \in C^{\infty}\left(H^{2}\right)$. Now let $f \in L^{\rho}\left(H^{2}\right)$ satisfying $f-h \in$ $X_{\mathbb{S}}$ for every $h \in C^{\infty}\left(H^{2}\right)$. Since $\mu\left(H^{2}\right)<\infty$ and $\rho$ is absolutely continuous and strongly finite, it is known that $\rho$ is absolutely finite on $X\left(H^{2}\right)$. Now by using these properties of the modular $\rho$, one can see from [5, 16] that the space $C^{\infty}\left(H^{2}\right)$ is modularly dense in $L^{\rho}\left(H^{2}\right)$, i.e. there exists a sequence $\left\{h_{l, n}\right\} \subset C^{\infty}\left(H^{2}\right)$ such that

$$
P-\lim _{l, n} \rho\left(3 \eta_{0}^{*}\left(h_{l, n}-f\right)\right)=0, \quad \text { for some } \eta_{0}>0
$$

By this, we have that for every $\epsilon>0$ there is a positive number $l_{0}=l_{0}(\epsilon)$ so that

$$
\rho\left(3 \eta_{0}^{*}\left(h_{l, n}-f\right)\right)<\epsilon \quad \text { for every } l, n \geq l_{0}
$$


Now since $S_{i, j}$ is a positive linear operator, we may write

$$
\begin{aligned}
\eta_{0}^{*}\left|\sum_{i, j=1,1}^{\infty, \infty} a_{k l i j} S_{i, j}(f ; y, z)-f(y, z)\right| \leq & \eta_{0}^{*}\left|\sum_{i, j=1,1}^{\infty, \infty} a_{k l i j} S_{i, j}\left(f-h_{l_{0}, l_{0}} ; y, z\right)\right| \\
& +\eta_{0}^{*}\left|\sum_{i, j=1,1}^{\infty, \infty} a_{k l i j} S_{i, j}\left(h_{l_{0}, l_{0}} ; y, z\right)-h_{l_{0}, l_{0}}(y, z)\right| \\
& +\eta_{0}^{*}\left|h_{l_{0}, l_{0}}(y, z)-f(y, z)\right|
\end{aligned}
$$

for every $y, z \in H$ and $i, j \in \mathbb{N}$. By applying the modular $\rho$ to both sides of the above inequality and using the monotonicity of $\rho$ and also multiplying both sides of the above inequality by $\frac{1}{|\sigma(y, z)|}$, we get

$$
\begin{aligned}
\rho\left(\eta_{0}^{*}\left(\frac{\sum_{i, j=1,1}^{\infty, \infty} a_{k l i j} S_{i, j} f-f}{\sigma(y, z)}\right)\right) \leq & \rho\left(3 \eta_{0}^{*} \frac{\sum_{i, j=1,1}^{\infty, \infty} a_{k l i j} S_{i, j}\left(f-h_{l_{0}, l_{0}}\right)}{\sigma(y, z)}\right) \\
& +\rho\left(3 \eta_{0}^{*} \frac{\sum_{i, j=1,1}^{\infty, \infty} a_{k l i j} S_{i, j} h_{l_{0}, l_{0}}-h_{l_{0}, l_{0}}}{\sigma(y, z)}\right) \\
& +\rho\left(3 \eta_{0}^{*}\left(\frac{h_{l_{0}, l_{0}}-f}{\sigma(y, z)}\right)\right) .
\end{aligned}
$$

By observing that $|\sigma(y, z)| \geq b>0\left(b=\max \left\{b_{m}: m=0,1,2,3\right\}\right)$, we can write

$$
\begin{aligned}
\rho\left(\eta_{0}^{*}\left(\frac{\sum_{i, j=1,1}^{\infty, \infty} a_{k l i j} S_{i, j} f-f}{\sigma(y, z)}\right)\right) \leq & \rho\left(3 \eta_{0}^{*} \frac{\sum_{i, j=1,1}^{\infty, \infty} a_{k l i j} S_{i, j}\left(f-h_{l_{0}, l_{0}}\right)}{\sigma(y, z)}\right) \\
& +\rho\left(3 \eta_{0}^{*} \frac{\sum_{i, j=1,1}^{\infty, \infty} a_{k l i j} S_{i, j} h_{l_{0}, l_{0}}-h_{l_{0}, l_{0}}}{\sigma(y, z)}\right) \\
& +\rho\left(\frac{3 \eta_{0}^{*}}{b}\left(h_{l_{0}, l_{0}}-f\right)\right) .
\end{aligned}
$$

By using the above inequality and 2.7), we get

$$
\begin{aligned}
\rho\left(\eta_{0}^{*}\left(\frac{\sum_{i, j=1,1}^{\infty, \infty} a_{k l i j} S_{i, j} f-f}{\sigma(y, z)}\right)\right) \leq \epsilon & +\rho\left(3 \eta_{0}^{*} \frac{\sum_{i, j=1,1}^{\infty, \infty} a_{k l i j} S_{i, j}\left(f-h_{l_{0}, l_{0}}\right)}{\sigma(y, z)}\right) \\
& +\rho\left(3 \eta_{0}^{*} \frac{\sum_{i, j=1,1}^{\infty, \infty} a_{k l i j} S_{i, j} h_{l_{0}, l_{0}}-h_{l_{0}, l_{0}}}{\sigma(y, z)}\right) .
\end{aligned}
$$


Hence, by using the fact that $h_{l_{0}, l_{0}} \in C^{\infty}\left(H^{2}\right)$ and $f-h_{l_{0}, l_{0}} \in X_{\mathbb{S}}$, we have

$$
\begin{gathered}
\frac{1}{q(c, d)} \sum_{i, j=0}^{\infty} q_{i, j} c^{i} d^{j} \rho\left(\eta_{0}\left(\frac{\sum_{i, j=1,1}^{\infty, \infty} a_{k l i j} S_{i, j} f-f}{\sigma(y, z)}\right)\right) \\
\leq \epsilon+\frac{1}{q(c, d)} \sum_{i, j=0}^{\infty} q_{i, j} c^{i} d^{j} \rho\left(3 \eta_{0}^{*} \frac{\sum_{i, j=1,1}^{\infty, \infty} a_{k l i j} S_{i, j}\left(f-h_{l_{0}, l_{0}}\right)}{q(c, d)} \sum_{i, j=0}^{\infty} q_{i, j} c^{i} d^{j} \rho\left(3 \eta_{0}^{*} \frac{\sum_{i, j=1,1}^{\infty, \infty} a_{k l i j} S_{i, j} h_{l_{0}, l_{0}}-h_{l_{0}, l_{0}}}{\sigma(y, z)}\right)\right.
\end{gathered}
$$

Taking limit superior as $c, d \rightarrow R^{-}$on both sides, and by using (1.4), we get

$$
\begin{aligned}
\limsup _{c, d \rightarrow R^{-}} & \frac{1}{q(c, d)} \sum_{i, j=0}^{\infty} q_{i, j} c^{i} d^{j} \rho\left(\eta_{0}\left(\frac{\sum_{i, j=1,1}^{\infty, \infty} a_{k l i j} S_{i, j} f-f}{\sigma(y, z)}\right)\right) \\
\leq & \epsilon+T \rho\left[3 \eta_{0}^{*}\left(f-h_{l_{0}, l_{0}}\right)\right] \\
& +\limsup _{c, d \rightarrow R^{-}} \frac{1}{q(c, d)} \sum_{i, j=0}^{\infty} q_{i, j} c^{i} d^{j} \rho\left(3 \eta_{0}^{*} \frac{\sum_{i, j=1,1}^{\infty, \infty} a_{k l i j} S_{i, j} h_{l_{0}, l_{0}}-h_{l_{0}, l_{0}}}{\sigma(y, z)}\right) .
\end{aligned}
$$

This implies that

$$
\begin{aligned}
& \limsup _{c, d \rightarrow R^{-}} \frac{1}{q(c, d)} \sum_{i, j=0}^{\infty} q_{i, j} c^{i} d^{j} \rho\left(\eta_{0}\left(\frac{\sum_{i, j=1,1}^{\infty, \infty} a_{k l i j} S_{i, j} f-f}{\sigma(y, z)}\right)\right) \\
& \leq \epsilon+T \epsilon+\limsup _{c, d \rightarrow R^{-}} \frac{1}{q(c, d)} \sum_{i, j=0}^{\infty} q_{i, j} c^{i} d^{j} \rho\left(3 \eta_{0}^{*} \frac{\sum_{i, j=1,1}^{\infty, \infty} a_{k l i j} S_{i, j} h_{l_{0}, l_{0}}-h_{l_{0}, l_{0}}}{\sigma(y, z)}\right) .
\end{aligned}
$$

By 2.3), we have

$$
\limsup _{c, d \rightarrow R^{-}} \frac{1}{q(c, d)} \sum_{i, j=0}^{\infty} q_{i, j} c^{i} d^{j} \rho\left(3 \eta_{0}^{*} \frac{\sum_{i, j=1,1}^{\infty, \infty} a_{k l i j} S_{i, j} h_{l_{0}, l_{0}}-h_{l_{0}, l_{0}}}{\sigma(y, z)}\right)=0
$$




$$
\Rightarrow \limsup _{c, d \rightarrow R^{-}} \frac{1}{q(c, d)} \sum_{i, j=0}^{\infty} q_{i, j} c^{i} d^{j} \rho\left(\eta_{0}\left(\frac{\sum_{i, j=1,1}^{\infty, \infty} a_{k l i j} S_{i, j} f-f}{\sigma(y, z)}\right)\right) \leq \epsilon+T \epsilon .
$$

As $\epsilon$ is an arbitrary positive real number, we have

$$
\limsup _{c, d \rightarrow R^{-}} \frac{1}{q(c, d)} \sum_{i, j=0}^{\infty} q_{i, j} c^{i} d^{j} \rho\left(3 \eta_{0}^{*} \frac{\sum_{i, j=1,1}^{\infty, \infty} a_{k l i j} S_{i, j} f-f}{\sigma(y, z)}\right)=0 .
$$

Moreover, $\frac{1}{q(c, d)} \sum_{i, j=0}^{\infty} q_{i, j} c^{i} d^{j} \rho\left(3 \eta_{0}^{*} \frac{\sum_{i, j=1,1}^{\infty, \infty} a_{k l i j} S_{i, j} f-f}{\sigma(y, z)}\right)$ is non negative, so we get

$$
\lim _{c, d \rightarrow R^{-}} \frac{1}{q(c, d)} \sum_{i, j=0}^{\infty} q_{i, j} c^{i} d^{j} \rho\left(3 \eta_{0}^{*} \frac{\sum_{i, j=1,1}^{\infty, \infty} a_{k l i j} S_{i, j} f-f}{\sigma(y, z)}\right)=0 .
$$

Hence the proof is finished.

Theorem 2.2. Suppose that $\rho, \sigma(y, z), A=\left(a_{k l i j}\right)$, and $\mathbb{S}=\left\{S_{i, j}\right\}$ are as in Theorem 2.1. If the modular $\rho$ satisfies the $\Delta_{2}$ condition, then the following statements are equivalent:

$$
\text { (i) } \lim _{c, d \rightarrow R^{-}} \frac{1}{q(c, d)} \sum_{i, j=0}^{\infty} q_{i, j} c^{i} d^{j} \rho\left(\eta\left(\frac{\sum_{i, j=1,1}^{\infty, \infty} a_{k l i j} S_{i, j} e_{m}-e_{m}}{\sigma_{m}(y, z)}\right)\right)=0
$$

for every $\eta>0$ and $m=0,1,2,3$.

(ii) $\lim _{c, d \rightarrow R^{-}} \frac{1}{q(c, d)} \sum_{i, j=0}^{\infty} q_{i, j} c^{i} d^{j} \rho\left(\eta\left(\frac{\sum_{i, j=1,1}^{\infty, \infty} a_{k l i j} S_{i, j} f-f}{\sigma(y, z)}\right)\right)=0$

for every $\eta>0$ provided that $f \in L^{\rho}\left(H^{2}\right)$ such that $f-g \in X_{\mathbb{S}}$ for every $g \in C^{\infty}\left(H^{2}\right)$.

Condition 1.4 reduces to

$$
\limsup _{c, d \rightarrow R^{-}} \frac{1}{q(c, d)} \sum_{i, j=0}^{\infty} q_{i, j} c^{i} d^{j} \rho\left(\eta\left(\sum_{i, j=1,1}^{\infty, \infty} a_{k l i j} S_{i, j} g\right)\right) \leq T \rho(\eta h)
$$

for every $g \in X_{\mathbb{S}}, \eta>0$ and for an absolute positive constant $T$, if one replaces the scale function by a non-zero constant.

Corollary 2.3. Let $\mathbb{S}=\left\{S_{i, j}\right\}$ be a double sequence of positive linear operators from $G$ into $X\left(H^{2}\right)$ satisfying (2.8), $A=\left(a_{k l i j}\right)$ be an identity matrix, $\rho$ be a strongly finite, monotone, absolutely continuous and $Q$-quasi convex modular on 
$X\left(H^{2}\right)$. If $\left\{S_{i, j} e_{m}\right\}$ is strongly convergent to $e_{m}$ in the sense of the power series, for each $m=0,1,2,3$, then $\left\{S_{i, j} f\right\}$ is modularly convergent to $f$ in the sense of the power series provided $f \in L^{\rho}\left(H^{2}\right)$ such that $f-h \in X_{\mathbb{S}}$ for every $h \in C^{\infty}\left(H^{2}\right)$.

Corollary 2.4. Let $\mathbb{S}=\left\{S_{i, j}\right\}, A=\left(a_{k l i j}\right)$ be an identity matrix, and $\rho$ be the same as in Corollary 2.3. If $\rho$ satisfies the $\Delta_{2}$ condition, then the following statements are equivalent:

(i) $\left\{S_{i, j} e_{m}\right\}$ is strongly convergent to $e_{m}$ in the sense of the power series method, for each $m=0,1,2,3$.

(ii) $\left\{S_{i, j} f\right\}$ is strongly convergent to $f$ in the sense of the power series method, for $f \in L^{\rho}\left(H^{2}\right)$ such that $f-h \in X_{\mathbb{S}}$ for every $h \in C^{\infty}\left(H^{2}\right)$.

If the power series convergence reduces to the Pringsheim convergence, then (1.4) becomes

$$
P-\limsup _{i, j} \rho\left(\eta\left(\frac{\sum_{i, j=1,1}^{\infty, \infty} a_{k l i j} S_{i, j} g}{\sigma(y, z)}\right)\right) \leq T \rho(\eta g)
$$

for every $g \in X_{\mathbb{S}}, \eta>0$ and for an absolute positive constant $T$.

Corollary 2.5. Let $\mathbb{S}=\left\{S_{i, j}\right\}$ be a double sequence of positive linear operators from $G$ into $X\left(H^{2}\right)$ satisfying $(2.9), A=\left(a_{k l i j}\right)$ be an identity matrix, $\rho$ be a strongly finite, monotone, absolutely continuous and $Q$-quasi convex modular on $X\left(H^{2}\right)$. Moreover, let $\sigma_{m}(y, z)$ be an unbounded function satisfying $\left|\sigma_{m}(y, z)\right| \geq$ $b_{m}>0$, for $m=0,1,2,3$. If $\left\{S_{i, j} e_{m}\right\}$ is relatively strongly convergent to $e_{m}$, for each $m=0,1,2,3$, then $\left\{S_{i, j} f\right\}$ is relatively modularly convergent to $f$ provided $f \in L^{\rho}\left(H^{2}\right)$ such that $f-h \in X_{\mathbb{S}}$ for every $h \in C^{\infty}\left(H^{2}\right)$.

Corollary 2.6. Let $\mathbb{S}=\left\{S_{i, j}\right\}, A=\left(a_{k l i j}\right), \rho$, and $\sigma_{m}(y, z)$, for $m=0,1,2,3$, be the same as in Corollary 2.5. If $\rho$ satisfies the $\Delta_{2}$ condition, then the following are equivalent:

(i) $\left\{S_{i, j} e_{m}\right\}$ is relatively strongly convergent to $e_{m}$, for each $m=0,1,2,3$.

(ii) $\left\{S_{i, j} f\right\}$ is relatively strongly convergent to $f$, for $f \in L^{\rho}\left(H^{2}\right)$ such that $f-h \in X_{\mathbb{S}}$ for every $h \in C^{\infty}\left(H^{2}\right)$.

Example 2.7. Let $\xi:[0, \infty) \rightarrow[0, \infty)$ be a continuous function such that the following conditions hold:

(i) $\xi$ is convex.

(ii) $\xi=0, \xi(v) \geq 0$ for $v>0$, and $\lim _{v \rightarrow \infty} \xi(v)=\infty$.

Consider $A=\left(a_{k l i j}\right)$ an identity matrix, $H=[0,1]$ and a functional $\rho^{\xi}$ on $X\left(H^{2}\right)$ defined by

$$
\rho^{\xi}(f)=\int_{0}^{1} \int_{0}^{1} \xi(|f(y, z)|) d y d z
$$

for $f \in X\left(H^{2}\right)$. Here $\rho^{\xi}$ is a convex modular on $X\left(H^{2}\right)$. Suppose that

$$
L_{\xi}^{\rho}\left(H^{2}\right)=\left\{f \in X\left(H^{2}\right): \rho^{\xi}(\eta f)<+\infty, \text { for some } \eta>0\right\}
$$


is an Orlicz space generated by $\xi$. Now consider the bivariate Bernstein-Kantorovich operator $\mathbb{U}=\left\{U_{i, j}\right\}$ on the space $L_{\xi}^{\rho}\left(H^{2}\right)$, which is defined as follows:

$U_{i j}(f ; y, z)=\sum_{m=0}^{i} \sum_{n=0}^{j} p_{m, n}^{(i, j)}(y, z)(i+1)(j+1) \times \int_{m /(i+1)}^{(m+1) /(i+1)} \int_{n /(j+1)}^{(n+1) /(j+1)} f(r, s) d s d r$

for $y, z \in H$, where $p_{m, n}^{(i, j)}(y, z)$ is defined by

$$
p_{m, n}^{(i, j)}(y, z)=\left(\begin{array}{c}
i \\
m
\end{array}\right)\left(\begin{array}{l}
j \\
n
\end{array}\right) y^{m} z^{n}(1-y)^{i-m}(1-z)^{j-n}
$$

and

$$
\sum_{m=0}^{i} \sum_{n=0}^{j} p_{m, n}^{(i, j)}(y, z)=1
$$

Note that the operator $U_{i, j}$ maps the Orlicz space $L_{\xi}^{\rho}\left(H^{2}\right)$ into itself. Since 2.10 is satisfied, we can use the Jensen inequality. Therefore, for every $f \in L_{\xi}^{\rho}\left(H^{2}\right)$, there is an absolute constant $D>0$ such that

$$
\rho^{\xi}\left(\frac{U_{i, j} f}{\sigma(y, z)}\right) \leq D \rho^{\xi}(f)
$$

as in the proof of Lemma 5.1 in 3 .

Now for $X_{U}=L_{\xi}^{\rho}\left(H^{2}\right)$, property 2.9 is satisfied and for any function $f \in$ $L_{\xi}^{\rho}\left(H^{2}\right)$ such that $f-h \in X_{U}$ for every $h \in C^{\infty}\left(H^{2}\right),\left\{U_{i, j} f\right\}$ is relatively modularly convergent to $f$. We define a sequence of positive linear operators $W=\left\{W_{i, j}\right\}$ on $L_{\xi}^{\rho}\left(H^{2}\right)$ by using the operator $U_{i, j}$ as follows:

$$
W_{i, j}(f ; y, z)=\left(1+h_{i, j}(y, z)\right) U_{i, j}(f ; y, z)
$$

for $f \in L_{\xi}^{\rho}\left(H^{2}\right),(y, z) \in[0,1] \times[0,1]$ and $i, j \in \mathbb{N}$, where $\left\{h_{i, j}(y, z)\right\}$ is a sequence of zeros and ones which is not modularly convergent but relatively modularly convergent to 0 in the sense of the power series method. By using [3, Lemma 5.1] for every $g \in X_{W}=L_{\xi}^{\rho}\left(H^{2}\right)$, all $\eta>0$ and for an absolute positive constant $F$, we get

$$
\limsup _{c, d \rightarrow R^{-}} \frac{1}{q(c, d)} \sum_{i, j=0}^{\infty} q_{i, j} c^{i} d^{j} \rho^{\xi}\left(\eta \frac{W_{i, j} g}{\sigma(y, z)}\right) \leq F \rho^{\xi}(2 \eta g)
$$


Observe that

$$
\begin{aligned}
W_{i, j}\left(e_{0} ; y, z\right) & =\left(1+h_{i, j}(y, z)\right) \\
W_{i, j}\left(e_{1} ; y, z\right) & =\left(1+h_{i, j}(y, z)\right)\left(\frac{i y}{i+1}+\frac{1}{2(i+1)}\right), \\
W_{i, j}\left(e_{2} ; y, z\right) & =\left(1+h_{i, j}(y, z)\right)\left(\frac{j z}{j+1}+\frac{1}{2(j+1)}\right), \\
W_{i, j}\left(e_{3} ; y, z\right) & =\left(1+h_{i, j}(y, z)\right)\left(\frac{i(i-1) y^{2}}{(i+1)^{2}}+\frac{2 i y}{(i+1)^{2}}+\frac{1}{3(i+1)^{2}}\right. \\
& \left.+\frac{j(j-1) z^{2}}{(j+1)^{2}}+\frac{2 j z}{(j+1)^{2}}+\frac{1}{3(j+1)^{2}}\right) .
\end{aligned}
$$

So for any $\eta>0$ we have

$$
\begin{aligned}
\eta\left|\frac{W_{i, j}\left(e_{0} ; y, z\right)-e_{0}(y, z)}{\sigma(y, z)}\right| & =\eta\left(\frac{\left|1+h_{i, j}(y, z)-1\right|}{\sigma(y, z)}\right) \\
& =\eta\left(\frac{h_{i, j}(y, z)}{\sigma(y, z)}\right) .
\end{aligned}
$$

This implies

$$
\begin{aligned}
\rho^{\xi}\left[\eta\left(\frac{W_{i, j}\left(e_{0} ; y, z\right)-e_{0}(y, z)}{\sigma(y, z)}\right)\right] & =\rho^{\xi}\left(\eta \frac{h_{i, j}(y, z)}{\sigma(y, z)}\right) \\
& =\int_{0}^{1} \int_{0}^{1} \xi\left(\eta \frac{h_{i, j}(y, z)}{\sigma(y, z)}\right) d y d z \\
& =\xi\left(\eta \frac{h_{i, j}(y, z)}{\sigma(y, z)}\right) \\
& =\frac{h_{i, j}(y, z)}{\sigma(y, z)} \xi(\eta) .
\end{aligned}
$$

Since $\left\{h_{i, j}(y, z)\right\}$ converges relatively modularly to 0 in the sense of the power series method, for every $\eta>0$, we have

$$
\begin{aligned}
\limsup _{c, d \rightarrow R^{-}} & \frac{1}{q(c, d)} \sum_{i, j=0}^{\infty} q_{i, j} c^{i} d^{j} \rho^{\xi}\left(\eta\left(\frac{W_{i, j}\left(e_{0}\right)-e_{0}}{\sigma(y, z)}\right)\right) \\
& =\limsup _{c, d \rightarrow R^{-}} \frac{1}{q(c, d)} \sum_{i, j=0}^{\infty} q_{i, j} c^{i} d^{j} \frac{h_{i, j}(y, z)}{\sigma(y, z)} \xi(\eta) \\
& =0 .
\end{aligned}
$$

Moreover,

$$
\begin{aligned}
\eta\left|\frac{W_{i, j}\left(e_{1} ; y, z\right)-e_{1}(y, z)}{\sigma(y, z)}\right| & =\eta\left|\frac{y\left(\frac{i}{i+1}+\frac{i h_{i, j}(y, z)}{i+1}-1\right)+\frac{1}{2(i+1)}+\frac{h_{i, j}(y, z)}{2(i+1)}}{\sigma(y, z)}\right| \\
& \leq \eta\left\{\frac{\frac{3}{2(i+1)}+h_{i, j}(y, z)\left(\frac{2 i+1}{2(i+1)}\right)}{\sigma(y, z)}\right\} .
\end{aligned}
$$


Thus,

$$
\begin{aligned}
\rho^{\xi}\left[\eta\left(\frac{W_{i, j}\left(e_{1} ; y, z\right)-e_{1}(y, z)}{\sigma(y, z)}\right)\right] & \leq \rho^{\xi}\left(\eta\left\{\frac{\frac{3}{2(i+1)}+h_{i, j}(y, z)\left(\frac{2 i+1}{2(i+1)}\right)}{\sigma(y, z)}\right\}\right) \\
& \leq \frac{h_{i, j}(y, z)}{\sigma(y, z)} \rho^{\xi}\left[\eta\left(\frac{2 i+1}{i+1}\right)\right]+\frac{\rho^{\xi}\left(\eta \frac{3}{(i+1)}\right)}{\sigma(y, z)} .
\end{aligned}
$$

As $\left\{\frac{2 i+1}{i+1}\right\}$ is convergent, there exists a constant $M>0$ such that $\left\{\frac{2 i+1}{i+1} \leq M\right\}$ for every $i \in \mathbb{N}$. Then by using the monotonicity of $\rho^{\xi}$, for any $\eta>0$ we have

$$
\rho^{\xi}\left[\eta\left(\frac{2 i+1}{i+1}\right)\right] \leq \rho^{\xi}(\eta M) .
$$

This implies

$$
\begin{aligned}
\rho^{\xi}\left[\eta\left(\frac{W_{i, j}\left(e_{1}\right)-e_{1}}{\sigma(y, z)}\right)\right] & \leq \frac{h_{i, j}(y, z)}{\sigma(y, z)} \rho^{\xi}(\eta M)+\frac{\rho^{\xi}\left(\frac{3 \eta}{(i+1)}\right)}{\sigma(y, z)} \\
& =\frac{h_{i, j}(y, z)}{\sigma(y, z)} \xi(\eta M)+\frac{\xi\left(\frac{3 \eta}{(i+1)}\right)}{\sigma(y, z)} .
\end{aligned}
$$

Since $\xi$ is continuous, we have

$$
\lim _{i} \xi\left(\frac{3 \eta}{(i+1)}\right)=\xi\left(\lim _{i} \frac{3 \eta}{(i+1)}\right)=\xi(0)=0
$$

Hence, we get that $\frac{\xi\left(\frac{3 \eta}{(i+1)}\right)}{\sigma(y, z)}$ is convergent to 0 in the sense of the power series method. Therefore,

$$
\begin{aligned}
\limsup _{c, d \rightarrow R^{-}} & \frac{1}{q(c, d)} \sum_{i, j=0}^{\infty} q_{i, j} c^{i} d^{j} \rho^{\xi}\left(\frac{\eta W_{i, j}\left(e_{1}\right)-e_{1}}{\sigma(y, z)}\right) \\
\leq & \limsup _{c, d \rightarrow R^{-}} \frac{1}{q(c, d)} \sum_{i, j=0}^{\infty} q_{i, j} c^{i} d^{j}\left[\frac{h_{i, j}(y, z)}{\sigma(y, z)} \xi(\eta M)+\frac{3 \eta}{(i+1)}\right) \\
= & \xi(\eta M) \limsup _{c, d \rightarrow R^{-}} \frac{1}{q(c, d)} \sum_{i, j=0}^{\infty} q_{i, j} c^{i} d^{j} \frac{h_{i, j}(y, z)}{\sigma(y, z)} \\
& +\limsup _{c, d \rightarrow R^{-}} \frac{1}{q(c, d)} \sum_{i, j=0}^{\infty} q_{i, j} c^{i} d^{j} \frac{\xi\left(\frac{3 \eta}{(i+1)}\right)}{\sigma(y, z)} \\
= & 0 .
\end{aligned}
$$


Similarly, we have $\limsup _{c, d \rightarrow R^{-}} \frac{1}{q(c, d)} \sum_{i, j=0}^{\infty} q_{i, j} c^{i} d^{j} \rho^{\xi}\left(\frac{\eta W_{i, j}\left(e_{2}\right)-e_{2}}{\sigma(y, z)}\right)=0$.

Finally,

$$
\begin{aligned}
\eta \mid & \frac{W_{i, j}\left(e_{3} ; y, z\right)-e_{3}(y, z)}{\sigma(y, z)} \mid \\
= & \frac{\eta}{\sigma(y, z)} \mid \frac{y^{2} i(i-1)}{(i+1)^{2}}+\frac{2 i y}{(i+1)^{2}}+\frac{1}{3(i+1)^{2}}+\frac{z^{2} j(j-1)}{(j+1)^{2}}+\frac{2 j z}{(j+1)^{2}}+\frac{1}{3(j+1)^{2}} \\
& +h_{i, j}(y, z) \frac{i(i-1)}{(i+1)^{2}} y^{2}+\frac{2 i y h_{i, j}(y, z)}{(i+1)^{2}}+\frac{h_{i, j}(y, z)}{3(i+1)^{2}}+\frac{j(j-1)}{(j+1)^{2}} z^{2} h_{i, j}(y, z) \\
& +\frac{2 i z h_{i, j}(y, z)}{(j+1)^{2}}+\frac{h_{i, j}(y, z)}{3(j+1)^{2}}-y^{2}-z^{2} \mid \\
= & \frac{\eta}{\sigma(y, z)} \mid y^{2}\left[\frac{i(i-1)}{(i+1)^{2}}+\frac{h_{i, j}(y, z) i(i-1)}{(i+1)^{2}}-1\right]+y\left[\frac{2 i}{(i+1)^{2}}+\frac{h_{i, j}(y, z) 2 i}{(i+1)^{2}}\right] \\
& +z^{2}\left[\frac{j(j-1)}{(j+1)^{2}}+\frac{h_{i, j}(y, z) j(j-1)}{(j+1)^{2}}-1\right]+z\left[\frac{2 j}{(j+1)^{2}}+\frac{h_{i, j}(y, z) 2 j}{(j+1)^{2}}\right]+\frac{1}{3(i+1)^{2}} \\
& +\frac{1}{3(j+1)^{2}}+\frac{h_{i, j}(y, z)}{3(i+1)^{2}}+\frac{h_{i, j}(y, z)}{3 j(i+1)^{2}} \mid \\
= & \eta\left\{\frac{15 i+4}{\sigma(y, z) 3(i+1)^{2}}+\frac{15 j+4}{\sigma(y, z) 3(j+1)^{2}}+\frac{h_{i, j}(y, z)}{\sigma(y, z)}\left[\frac{3 i^{2}+3 i+1}{3(i+1)^{2}}+\frac{3 j^{2}+3 j+1}{3(j+1)^{2}}\right]\right\} .
\end{aligned}
$$

Since $\left\{\frac{3 i^{2}+3 i+1}{3(i+1)^{2}}\right\}$ and $\left\{\frac{3 j^{2}+3 j+1}{3(j+1)^{2}}\right\}$ are convergent, there exist constants $X, Z>0$ such that $\left|\frac{3 i^{2}+3 i+1}{3(i+1)^{2}}\right| \leq X$, for every $i \in \mathbb{N}$, and $\left|\frac{3 j^{2}+3 j+1}{3(j+1)^{2}}\right| \leq Z$, for every $j \in \mathbb{N}$. Now by using the definition of $\left\{h_{i, j}(y, z)\right\}$ and by the monotonicity of $\rho^{\xi}$, we have

$$
\begin{aligned}
& \rho^{\xi}\left[\eta\left(\frac{W_{i, j}\left(e_{3} ; y, z\right)-e_{3}(y, z)}{\sigma(y, z)}\right)\right] \\
& \quad \leq \rho^{\xi}\left[\frac{2 \eta\left(\frac{15 i+4}{3(i+1)^{2}}\right)+2 \eta\left(\frac{15 j+4}{3(j+1)^{2}}\right)}{\sigma(y, z)}\right]+\rho^{\xi}\left[\frac{2 \eta h_{i, j}(y, z)\left[\frac{3 i^{2}+3 i+1}{3(i+1)^{2}}+\frac{3 j^{2}+3 j+1}{3(j+1)^{2}}\right]}{\sigma(y, z)}\right] \\
& \quad \leq \rho^{\xi}\left[\frac{\eta\left(\frac{30 i+8}{3(i+1)^{2}}\right)+\eta\left(\frac{30 j+8}{3(j+1)^{2}}\right)}{\sigma(y, z)}\right]+\rho^{\xi}\left[\frac{2 \eta h_{i, j}(y, z)(X+Z)}{\sigma(y, z)}\right] .
\end{aligned}
$$

This implies

$$
\begin{aligned}
& \rho^{\xi}\left[\eta\left(\frac{W_{i, j}\left(e_{3} ; y, z\right)-e_{3}(y, z)}{\sigma(y, z)}\right)\right] \leq \frac{\xi\left(\eta\left[\frac{30 i+8}{3(i+1)^{2}}+\frac{30 j+8}{3(j+1)^{2}}\right]\right)}{\sigma(y, z)} \\
& +\frac{h_{i, j}(y, z)}{\sigma(y, z)} \xi(2 \eta[X+Z]) .
\end{aligned}
$$


Since $\xi$ is continuous, we have

$$
\begin{aligned}
\lim _{i, j \rightarrow \infty} \xi\left(\eta\left[\frac{30 i+8}{3(i+1)^{2}}+\frac{30 j+8}{3(j+1)^{2}}\right]\right) & =\xi\left(\eta \lim _{i, j \rightarrow \infty}\left[\frac{30 i+8}{3(i+1)^{2}}+\frac{30 j+8}{3(j+1)^{2}}\right]\right) \\
& =\xi(0) \\
& =0 .
\end{aligned}
$$

Thus,

$$
\frac{\xi\left(\eta\left[\frac{30 i+8}{3(i+1)^{2}}+\frac{30 j+8}{3(j+1)^{2}}\right]\right)}{\sigma(y, z)}
$$

is convergent to 0 in the sense of the power series method. So by using this and by the definition of $\left\{h_{i, j}(y, z)\right\}$ we have

$$
\limsup _{c, d \rightarrow R^{-}} \frac{1}{q(c, d)} \sum_{i, j=0}^{\infty} q_{i, j} c^{i} d^{j} \rho^{\xi}\left(\eta\left(\frac{W_{i, j}\left(e_{3}\right)-e_{3}}{\sigma(y, z)}\right)\right)=0 \quad \text { for every } \eta>0 .
$$

Hence, we can conclude that the sequence $W=\left\{W_{i, j}\right\}$ satisfies all the assumptions of Theorem 2.1. Therefore,

$$
\limsup _{c, d \rightarrow R^{-}} \frac{1}{q(c, d)} \sum_{i, j=0}^{\infty} q_{i, j} c^{i} d^{j} \rho^{\xi}\left(\eta_{0}\left(\frac{W_{i, j}(f)-f}{\sigma(y, z)}\right)\right)=0 \quad \text { for every } \eta_{0}>0
$$

holds for every $f \in L_{\xi}^{\rho}\left(H^{2}\right)$ such that $f-h \in X_{W}$ for every $h \in C^{\infty}\left(H^{2}\right)$.

Remark 2.8. (i) For $R=1, q(c, d)=\frac{1}{(1-c)} \frac{1}{(1-d)}$ and $i, j \geq 0, q_{i, j}=1$, the power series method coincides with Abel's method.

(ii) For $R=\infty, q(c, d)=e^{c} e^{d}$ and for $i, j \geq 0, q_{i, j}=\frac{1}{i !} \frac{1}{j !}$, the power series method coincides with Borel's method.

So we can prove all the theorems of this paper for Abel and Borel convergence.

\section{ACKNOWLEDGEMENT}

The authors would like to express their sincere thanks to the referee for his/her careful reading and several constructive comments which improved the presentation of the paper.

\section{REFERENCES}

[1] A. Alotaibi, M. Mursaleen and S. A. Mohiuddine, Korovkin type approximation theorem for $\sigma$-convergence of double sequences, J. Nonlinear Convex Anal. 16 (2015), no. 1, 183-192. MR 3315350

[2] C. Bardaro, A. Boccuto, K. Demirci, I. Mantellini and S. Orhan, Korovkin-type theorems for modular $\Psi$-A-statistical convergence, J. Funct. Spaces 2015 Art. ID 160401, 11 pp. MR 3310460

[3] C. Bardaro and I. Mantellini, Korovkin theorem in modular spaces, Comment. Math. (Prace Mat.) 47 (2007), no. 2, 239-253. MR 2377960

[4] C. Bardaro and I. Mantellini, A Korovkin theorem in multivariate modular function spaces, J. Funct. Spaces Appl. 7 (2009), no. 2, 105-120. MR 2541228. 
[5] C. Bardaro, J. Musielak and G. Vinti, Nonlinear Integral Operators and Applications, De Gruyter Series in Nonlinear Analysis and Applications 9, Walter de Gruyter, Berlin, 2003. MR 1994699

[6] C. Bardaro, A. Boccuto, X. Dimitriou and I. Mantellini, Abstract Korovkin-type theorems in modular spaces and applications, Cent. Eur. J. Math. 11 (2013), no. 10, 1774-1784. MR 3080236

[7] C. Belen, M. Mursaleen, and M. Yildirim, Statistical A-sumability of double sequences and a Korovkin type approximation theorem, Bull. Korean Math. Soc. 49 (2012), no. 4, 851-861. MR 2978422

[8] T. J. Bromwich, An introduction to the theory of infinite series, 2nd edition, Macmillan, London, 1965.

[9] E. W. Chittenden, Relatively uniform convergence of sequences of functions, Trans. Amer. Math. Soc. 15 (1914), no. 2, 197-201. MR 1500972

[10] E. W. Chittenden, On the limit functions of sequences of continuous functions converging relatively uniformly, Trans. Amer. Math. Soc. 20 (1919), no. 2, 179-184. MR 1501120.

[11] E. W. Chittenden, Relatively uniform convergence and the classification of functions, Trans. Amer. Math. Soc. 23 (1922), no. 1, 1-15. MR 1501185.

[12] K. Demirci and S. Orhan, Statistical relative approximation on modular spaces, Results Math. 71 (2017), no. 3-4, 1167-1184. MR 3648467.

[13] H. J. Hamilton, Transformations of multiple sequences, Duke Math. J. 2 (1936), no. 1, 29-60. MR 1545904

[14] G. H. Hardy, On the convergence of certain multiple series, Proc. Lond. Math. Soc. (2) 1 (1904), no. 1, 124-128.

[15] S. Karakuş and K. Demirci, Matrix summability and Korovkin type approximation theorem on modular spaces, Acta Math. Univ. Comenian. (N.S.) 79 (2010), no. 2, 281-292. MR 2745177

[16] I. Mantellini, Generalized sampling operators in modular spaces, Comment. Math. (Prace Mat.) 38 (1998), 77-92. MR 1672252.

[17] E. H. Moore, An Introduction to a Form of General Analysis, The New Haven Mathematical Colloquium. Yale University Press, New Haven, 1910.

[18] F. Móricz, Extensions of the spaces $c$ and $c_{0}$ from single to double sequences, Acta Math. Hungar. 57 (1991), no. 1-2, 129-136. MR 1128849

[19] F. Móricz and B. E. Rhoades, Almost convergence of double sequences and strong regularity of summability matrices, Math. Proc. Cambridge Philos. Soc. 104 (1988), no. 2, 283-294. MR 0948914

[20] M. Mursaleen and S. A. Mohiuddine, Korovkin type approximation theorem for almost and statistical convergence, Nonlinear Analysis, 487-494. Springer Optim. Appl., 68, Springer, New York, 2012. MR 2962656.

[21] M. Mursaleen and A. Alotaibi, Korovkin type approximation theorem for functions of two variables through statistical A-summability, Adv. Difference Equ. 2012, 2012:65, 10 pp. MR 2948728

[22] S. Orhan and K. Demirci, Statistical approximation by double sequences of positive linear operators on modular spaces, Positivity 19 (2015), no. 1, 23-36. MR 3325319

[23] S. Orhan and K. Demirci, Statistical $\mathcal{A}$-summation process and Korovkin type approximation theorem on modular spaces, Positivity 18 (2014), no. 4, 669-686. MR 3275359

[24] A. Pringsheim, Zur Theorie der zweifach unendlichen Zahlenfolgen, Math. Ann. 53 (1900), no. 3, 289-321. MR 1511092

[25] K. Raj, A. Choudhary and C. Sharma, Almost strongly Orlicz double sequence spaces of regular matrices and their applications to statistical convergence, Asian-Eur. J. Math. 11 (2018), no. 5, 1850073, 14 pp. MR 3869867.

[26] K. Raj and R. Anand, Double difference spaces of almost null and almost convergent sequences for Orlicz function, J. Comput. Anal. Appl. 24 (2018), no. 4, 773-783. MR 3752559. 
[27] G. M. Robison, Divergent double sequences and series, Trans. Amer. Math. Soc. 28 (1926), no. 1, 50-73. MR 1501332

[28] E. Taş, Some results concerning Mastroianni operators by power series method, Commun. Fac. Sci. Univ. Ank. Sér. A1 Math. Stat. 65 (2016), no. 1, 187-195. MR 3528890

[29] B. C. Tripathy, Statistically convergent double sequences, Tamkang J. Math. 34 (2003), no. 3, 231-237. MR 2001918

[30] B. Yılmaz, K. Demirci and S. Orhan, Relative modular convergence of positive linear operators, Positivity 20 (2016), no. 3, 565-577. MR 3540511.

[31] T. Yurdakadim, Some Korovkin type results via power series method in modular spaces, Commun. Fac. Sci. Univ. Ank. Sér. A1 Math. Stat. 65 (2016), no. 2, 65-76. MR 3528897

\author{
Kuldip Raj ${ }^{凶}$ \\ School of Mathematics, Shri Mata Vaishno Devi University, Katra-182320, J\&K, India \\ kuldipraj68@gmail.com
}

Anu Choudhary

School of Mathematics, Shri Mata Vaishno Devi University, Katra-182320, J\&K, India anuchoudhary407@gmail.com

Received: December 6, 2017

Accepted: October 11, 2018 